Radiologe $2022 \cdot 62: 130-139$

https://doi.org/10.1007/s00117-021-00955-8

Angenommen: 9. Dezember 2021

Online publiziert: 7. Januar 2022

(c) The Author(s), under exclusive licence to Springer Medizin Verlag $\mathrm{GmbH}$, ein Teil von Springer Nature 2021

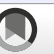

\title{
Röntgenbefunde bei diffusen parenchymatösen Lungenerkrankungen
}

Daria Kifjak ${ }^{1,2,3}$ - Johannes Leitner ${ }^{1,2} \cdot$ Raphael Ambros ${ }^{1,2} \cdot$ Benedikt H. Heidinger ${ }^{1,2}$. Ruxandra-Iulia Milos ${ }^{1,2} \cdot$ Lucian Beer $^{1,2} \cdot$ Florian Prayer $^{1,2} \cdot$ Sebastian Röhrich ${ }^{1,2}$. Helmut Prosch ${ }^{1,2}$

${ }^{1}$ Department of Biomedical Imaging and Image-guided Therapy, Medizinische Universität Wien, Wien, Österreich

${ }^{2}$ Universitätsklinik für Radiologie und Nuklearmedizin, Medizinische Universität Wien, Wien, Österreich

${ }^{3}$ Department of Radiology, University of Massachusetts Chan Medical School, Worcester, USA

\section{Zusammenfassung}

Klinisches Problem: Diffuse parenchymatöse Lungenerkrankungen umfassen eine heterogene Krankheitsgruppe des Lungenparenchyms, der Alveolarräume, der Gefäße sowie Atemwege, welche durch diverse Pathomechanismen, wie Entzündung sowie fibrotische Veränderungen, ausgelöst werden können. Da sich die Therapieansätze sowie Prognosen zwischen den Erkrankungen wesentlich unterscheiden, ist die korrekte Diagnosestellung von grundlegender Bedeutung. In der klinischen Routine spielt neben der Anamnese, der Klinik, den Laborbefunden und der Bronchoskopie die Bildgebung eine zentrale Rolle in der Diagnosefindung.

Empfehlungen für die Praxis: Die Diagnose diffuser parenchymatöser Lungenerkrankungen stellt eine enorme Herausforderung sowohl für Kliniker, Radiologen als auch Pathologen dar und sollte daher bevorzugt im multidisziplinären Rahmen erfolgen. Da sich die Patienten häufig mit einer unspezifischen, respiratorischen Symptomatik präsentieren, ist das Thorax-Röntgen die erste bildgebende Methode, welche eingesetzt wird. Bereits hier sind oft die verschiedenen Muster diffuser parenchymatöser Lungenerkrankungen (z. B. Milchglasverdichtungen und Konsolidierungen), deren Verteilung (z. B. kranial-kaudal) sowie zusätzliche Befunde, wie mediastinale Lymphadenopathie, bereits erkennbar. Der bildgebende Referenzstandard und somit integraler Bestandteil bei der Beurteilung einer diffusen parenchymatösen Lungenerkrankung ist jedoch die hochauflösende („high resolution“, HR) Computertomographie (CT) des Thorax. In manchen Fällen ist das Muster der HR-CT pathognomonisch, in anderen jedoch unspezifisch für eine Erkrankung, sodass weitere diagnostische Schritte nötig sind.

\section{Schlüsselwörter}

Thoraxröntgen · Idiopathische interstitielle Pneumonien · Sarkoidose · Langerhans-ZellHistiozytose $\cdot$ Hochauflösende Computertomographie

\section{Hintergrund}

Diffuse parenchymatöse Lungenerkrankungen (oft auch als interstitielle Lungenerkarkungen bezeichnet) umfassen eine heterogene Gruppe von mehr als 200 Erkrankungen des Lungenparenchyms, die sowohl vom pulmonalen Interstitium, den Alveolarräumen, den kleinen Atemwegen oder Gefäßen ausgehen können [1]. Die genaue Diagnose dieser Erkrankungen ist auf Grund der Vielfalt an Differentialdiagnosen, verbunden mit der Seltenheit der einzelnen Erkrankungen, eine besondere Herausforderung. Sie beruht neben der Anamnese, Klinik, Laborbefunden und Bronchoskopie zu einem großen Teil auf der Bildgebung [1, 2].

Das bildgebende Verfahren der Wahl ist zweifelsfrei die hochauflösende Computertomographie (high resolution CT, HRCT). Diese kann in vielen Fällen zur Dia- 
Tab. 1 Muster bei diffusen parenchymatösen Lungenerkrankungen mit den häufigsten Krankheitsbildern

\begin{tabular}{|c|c|}
\hline Muster & Erkrankungen \\
\hline \multicolumn{2}{|c|}{ Muster mit erhöhter Dichte } \\
\hline \multirow[t]{15}{*}{ Milchglas } & Akut: \\
\hline & $\begin{array}{l}\text { Infektionen/Pneumonien (Coronavirus disease } 2019 \text { (COVID-19), Cytomegalovi- } \\
\text { rus (CMV), Varizella Zoster Virus (VZV), Herpes Simplex Virus (HSV), Pneumocystis } \\
\text { jirovecii) }\end{array}$ \\
\hline & Aspiration \\
\hline & Lungenödem \\
\hline & Alveoläre Hämorrhagien (z. B. Antikoagulation, Vaskulitis) \\
\hline & Pneumonitis \\
\hline & Atemnotsyndrom des Erwachsenen (ARDS) \\
\hline & Medikamentenreaktionen \\
\hline & Chronisch: \\
\hline & Nicht Spezifische Interstitielle Pneumonie (NSIP) \\
\hline & Desquamative Interstitielle Pneumonie (DIP) \\
\hline & Exogen Allergische Alveolitis (EAA) \\
\hline & Organsierende Pneumonie (OP) \\
\hline & Chronische Eosinophile Pneumonie (CEP) \\
\hline & Alveolarproteinose \\
\hline \multirow{6}{*}{$\begin{array}{l}\text { Konsolidie- } \\
\text { rungen }\end{array}$} & Infektionen/Pneumonien \\
\hline & Organisierende Pneumonie (OP) \\
\hline & Chronisch eosinophile Pneumonie (CEP) \\
\hline & Lymphoproliferative Erkrankungen (z. B. Lymphome) \\
\hline & Lungenkarzinom, Metastasen \\
\hline & Fremdkörper \\
\hline \multicolumn{2}{|c|}{ Muster mit verminderter Dichte } \\
\hline Emphysem & COPD, alpha-1-Antitrypsinmangel \\
\hline \multirow[t]{7}{*}{ Zysten } & $\begin{array}{l}\text { Gewöhnliche Interstitielle Pneumonie (UIP)/Idiopathische Pulmonale Fibrose } \\
\text { (IPF) (Honeycombing) }\end{array}$ \\
\hline & Pneumozystis jirovecii Pneumonie \\
\hline & Langerhans-Zell-Histiozytose (LCH) \\
\hline & Lymphozytische Interstitielle Pneumonie (LIP) \\
\hline & Birt-Hogg-Dubé Syndrom \\
\hline & Lymphangioleiomyomatose (LAM) \\
\hline & Zystische Metastasen \\
\hline \multirow[t]{4}{*}{ Kavernen } & $\begin{array}{l}\text { Infektionen (Streptococcus, Staphylococcus aureus, Klebsiella, Haemophilus in- } \\
\text { fluenzae, Mycobacterium tuberculosis); typische und atypische Mycobacterien; } \\
\text { Pilze (Aspergillus, Pneumocystis jirovecii); Parasiten }\end{array}$ \\
\hline & Lungenabszess \\
\hline & Septische Emboli \\
\hline & Granulomatose mit Polyangiitis \\
\hline \multirow{9}{*}{$\begin{array}{l}\text { Noduläres } \\
\text { Muster }\end{array}$} & Sarkoidose \\
\hline & Infektionen (Pilze, Miliartuberkulose) \\
\hline & Respiratorische Bronchiolitis (RB) \\
\hline & Exogen Allergische Alveolitis (EAA) \\
\hline & Langerhans-Zell-Histiozytose (LCH) \\
\hline & $\begin{array}{l}\text { Pneumonien (Cytomegalovirus (CMV), Influenza, Parainfluenza-Virus, Masern, } \\
\text { Respiratorisches Synzytial-Virus (RSV)) }\end{array}$ \\
\hline & Pneumokoniosen (Silikose, Talkose, Beryllium-induziert) \\
\hline & Malignome (disseminiertes, primäres Lungenkarzinom, Metastasen, Lymphom) \\
\hline & Lungenhämosiderose \\
\hline
\end{tabular}

gnose einer Erkrankung führen, oder zumindest die Differentialdiagnose einengen. Die HR-CT ist eine in tiefer Inspiration durchgeführte $\mathrm{CT}$ ohne Kontrastmittel mit Lungenrekonstruktionen mit einer Schichtdicke $\leq 1 \mathrm{~mm}$. Im Vergleich zu der HR-CT, ist die Wertigkeit von ThoraxRöntgen Untersuchungen zur Abklärung von diffusen parenchymatösen Lungenerkrankungen deutlich eingeschränkt. Dies ist vor allem der Projektionsradiographie, den daraus resultierenden Überlagerungseffekten, sowie dem relativ niedrigen Kontrast der Thorax-Röntgen Aufnahmen geschuldet. So sind beispielsweise bei der Lymphangioleiomyomatose (LAM) lineare Verdichtungen das vorherrschendes Muster im Thorax-Röntgen, wohingegen das vorherrschende Muster in der HR-CT diffuse Zysten sind [2]. Ferner gilt es zu bedenken, dass in bis zu $10 \%$ der Fälle eine Thorax-Röntgen Aufnahme vollkommen normal aussehen kann, obwohl eine diffuse Lungenerkrankung vorliegt $[2,3]$. Aus diesem Grund sollte man im Falle eines unauffälligen Thorax-Röntgen, bei weiterhin bestehendem klinischen Verdacht auf eine interstitielle Lungenerkrankung, eine HRCT anschließen [3].

\section{Systematischer Zugang}

Diffuse parenchymatöse Lungenerkrankungen können sich aufgrund ihrer komplexen Pathologie sehr vielfältig präsentieren. Umso wichtiger ist es, sich an eine strukturierte und praktikable Vorgehensweise bei der Erstellung der Befunde zu halten. Eine mögliche Vorgehensweise für die Befundung ist die folgende [4]:

1. Identifizierung des (der) vorliegenden Muster(s)

2. Beschreibung zusätzlicher Befunde

3. Identifizierung der Verteilung der Veränderungen

\section{Muster}

Die Identifizierung des vorherrschenden Musters ist in Zusammenschau mit den erhobenen klinischen Parametern der erste Schritt in Richtung einer Eingrenzung möglicher Differentialdiagnosen. Dabei wird zwischen vier Mustergruppen unterschieden: i) erhöhte Dichte (z. B. Milchglas, Konsolidierungen, Atelektasen), 


\begin{tabular}{|l|l|}
\hline Tab. 1 (Fortsetzung) \\
\hline Muster & Erkrankungen \\
\hline Retikuläres & Akut: \\
\cline { 2 - 3 } Muster & Lungenödem \\
\cline { 2 - 3 } & Pneumonien (viral, Chlamydien, Mykoplasmen) \\
\cline { 2 - 3 } & Transfusionssyndrom \\
\cline { 2 - 3 } & Chronisch: \\
\cline { 2 - 3 } & Idiopathische Pulmonale Fibrose (IPF) \\
\cline { 2 - 3 } & Kollagenosen \\
\cline { 2 - 3 } & Asbestose \\
\cline { 2 - 3 } & Strahleninduzierte Pneumonitis \\
\cline { 2 - 3 } & $\begin{array}{l}\text { Malignome (lymphogene Metastasierung, bronchovaskuläre Form des Kaposi- } \\
\text { Sarkoms) }\end{array}$ \\
\cline { 2 - 2 } & Sarkoidose \\
\hline
\end{tabular}

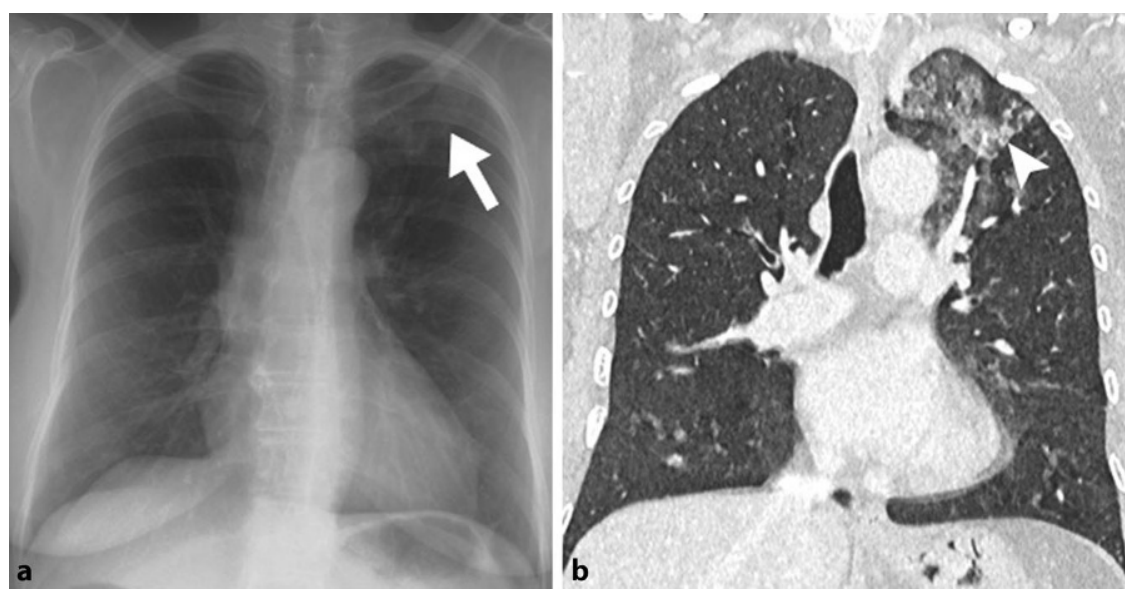

Abb. 1 \ 40 Jahre alte Patientin mit einer pulmonalen Einblutung in den linken Oberlappen. a Thoraxröntgen p/a: Milchglasartige Verschattung links apikal (weißer Pfeil), b HR-CT:Milchglasartige Verdichtung im Oberlappen links (weiße Pfeilspitze). HR-CT "high resolution“ Computertomographie

ii) verminderte Dichte (z. B. Emphysem, Zysten, Kavernen) iii) noduläre Muster und iv) retikuläre Muster ([3]; - Tab. 1).

\section{Milchglasartige Verdichtungen}

Milchglasverdichtungen sind im RöntgenThorax als Areal mit gering reduzierter Strahlentransparenz charakterisiert, im HR-CT als eine Anhebung der Dichte des Lungenparenchyms definiert, wobei die Bronchialwände und pulmonalen Gefäße weiterhin sichtbar bleiben ([3, 5]; -Abb. 1). Mögliche Gründe können einerseits eine inkomplette Ausfüllung der Alveolarräume (z.B. mit Sekret, Blut, Tumorzellen, Transsudat), andererseits ein partieller Kollaps von Alveolen oder eine Substanzvermehrung des Interstitiums bei Entzündung oder Fibrose sein $[3,6]$. Im Gegensatz zur HR-CT sind Milchverdichtungen im Röntgen-Thorax
Alveolitis (EAA) oder eine lymphoide interstitielle Pneumonie (LIP) $[3,6]$.

\section{Konsolidierungen}

Konsolidierungen sind definiert als eine Dichteanhebung des Lungenparenchyms mit fehlender Abgrenzbarkeit von Bronchialwänden und Gefäßen [3, 6]. Diese sind im Röntgen-Thorax als Transparenzminderungen zu sehen ([5]; • Abb. 2). Innerhalb kurzer Zeit neu-aufgetretene (akut) Konsolidierungen finden sich am häufigsten bei Pneumonien, einem alveolären Lungenödem, im Rahmen eines ARDS, Medikamentenreaktionen oder der akuten interstitiellen Pneumonie (AIP), akuten eosinophilen Pneumonien sowie der organisierenden Pneumonie (OP). Zu den Differentialdiagnosen chronischer Erkrankungen gehören beispielsweise die chronische $O P$, chronisch eosinophile Pneumonien (CEP), lymphoproliferative Erkrankungen (Lymphome) sowie Lungenkarzinome $[2,3]$.

\section{Noduläres Muster}

Das noduläre Muster besteht radiomorphologisch aus multiplen, kleinen Knötchen $(<1 \mathrm{~cm})$, welche anhand ihrer Größe, Dichte sowie Verteilung unterschieden werden können [3, 7]. Differentialdiagnostisch kommen beispielsweise die Sarkoidose, die Silikose, Malignome (z. B. miliar gestreute Metastasen), die respiratorische Bronchiolitis (RB) sowie Infektionen (z.B. Pilzinfektion, Miliartuberkulose) in Frage $[2,3,8]$. In vielen Fällen kann das Thorax-Röntgen normal aussehen, das HR-CT jedoch eine diffuse Beteiligung der gesamten Lunge zeigen, wie es bei der unkomplizierten RB oder in $90 \%$ der EAA [8]. Bei anderen Erkrankungen, wie beispielsweise der Silikose, kann das ThoraxRöntgen bereits frühe Veränderungen im Sinne kleiner, gut umschriebener, runder, oft verkalkter Knötchen, welche bevorzugt die posterioren, oberen Areale der Lungen betreffen, zeigen [8].

Im Gegensatz zur HR-CT, ist im Röntgenthorax eine Zuordnung von Noduli zu einem anatomischen Kompartiment (zentrilobulär, perilymphatisch oder zufällig) meist nicht möglich [9].

\section{Retikuläres Muster}

Der Begriff des retikulären Musters ist der Darstellung im Thorax-Röntgen geschul- 


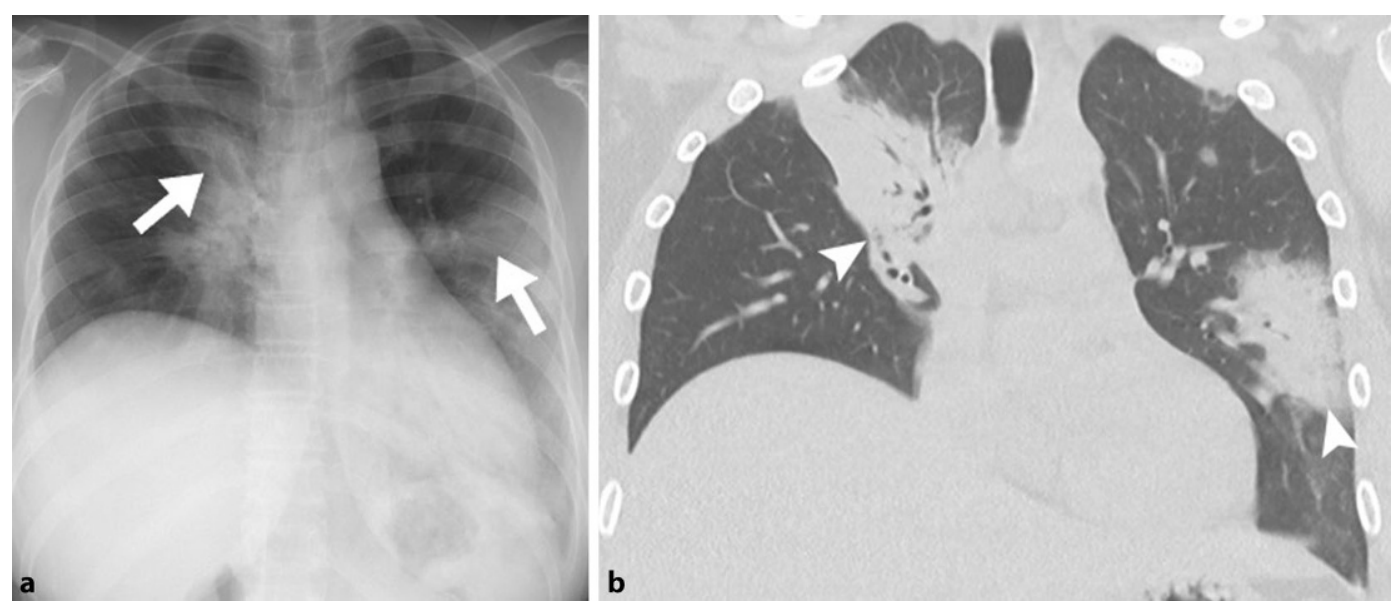

Abb. $2 \Delta 35$ Jahre alte Patientin mit histologisch nachgewiesener organisierender Pneumonie (OP). a Thoraxröntgen p/a: ausgedehnte Konsolidierungen parahilär im rechten sowie lateral im linken Mittelfeld (weiße Pfeile), b HR-CT: parahilär im rechten OL sowie peribronchial und lateral im linken OL verteilte Konsolidierungen mit pos. Aerobronchogramm (weiße Pfeilspitzen). HR-CT "high resolution“ Computertomographie, OL Oberlappen

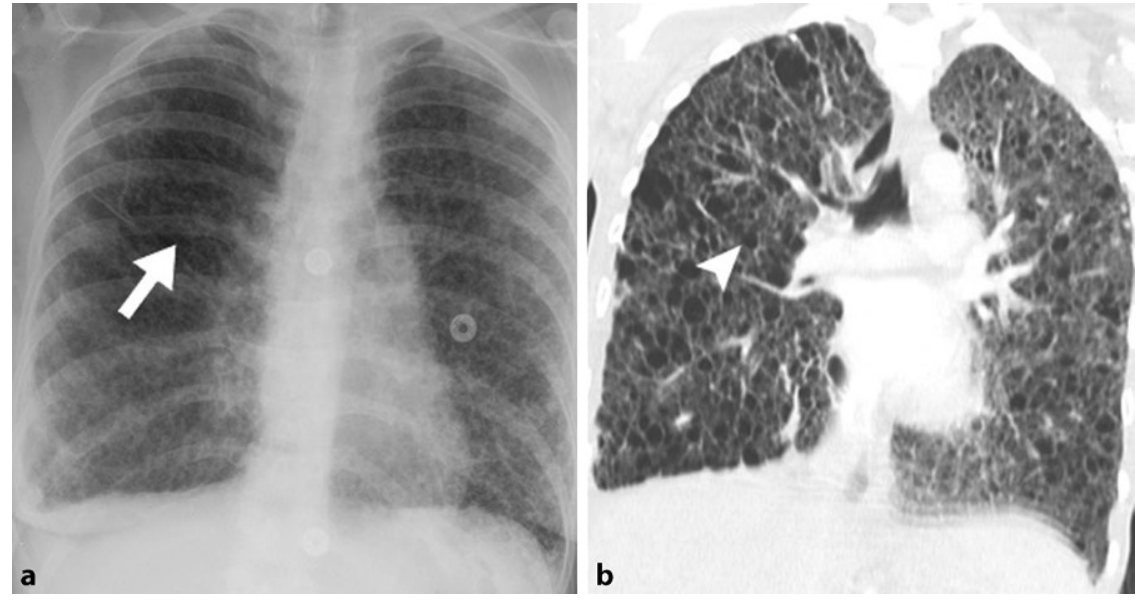

Abb. 3 \& 32 Jahre alte Patientin mit einer Lymphangioleiomyomatose (LAM). a Thoraxröntgen p/a: bilateral diffus verteiltes retikuläres Muster (weißerPfeil), b HR-CT: diffus, bilateral verteilte dünnwandige Zysten (weiße Pfeilspitze). HR-CT "high resolution“ Computertomographie
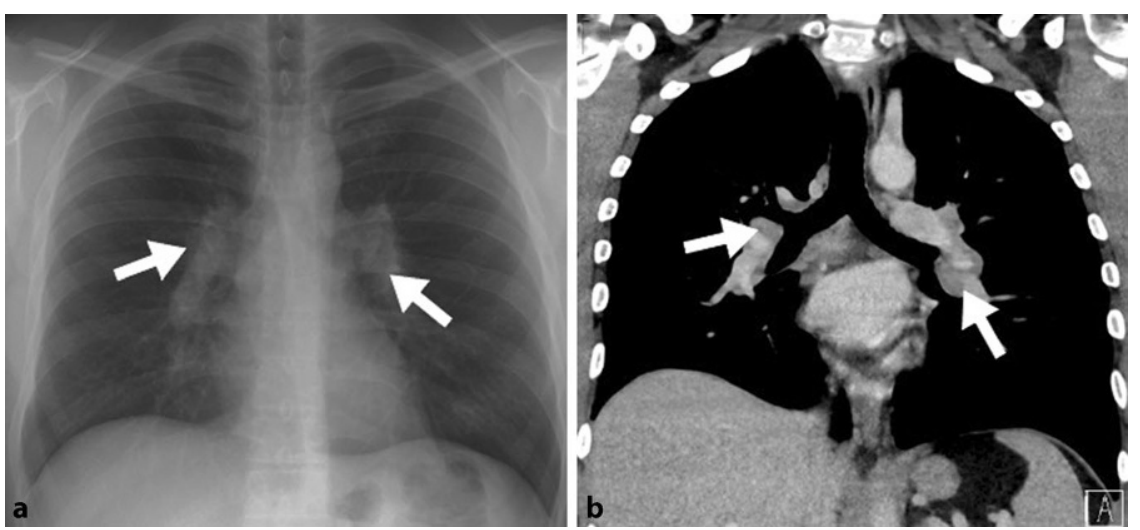

Abb. 4 \ 38 Jahre alter Patient mit bekannter Sarkoidose. a Thoraxröntgen p/a und b HR-CT: bilatrale, hiläre Lymphadenopathie (weiße Pfeile). HR-CT "high resolution“ Computertomographie det, das auf Grund von verflochtenen/sich überlappenden linearen Verdichtungen an ein Netz erinnert [2, 3]. Das Korrelat in der HR-CT sind beispielsweise verdickte interund intralobuläre Septen oder Wände von Zysten [10-12]. Es ist der häufigste radiologische Befund, welcher sich bei Patienten mit interstitiellen Pneumonien wiederfindet. In Kombination mit dem Honigwabenmuster zeigt es sich insbesondere bei der idiopathischen pulmonalen Fibrose (IPF) $[2,3,10]$. Ferner, kann in der HR-CT zwischen glatt und nodulär verdickten interlobulären Septumlinien unterschieden werden, wobei die glatt verdickten beispielsweise bei Lungenödemen, nodulär verdickte hingegen bei Sarkoidosen, vorkommen [7]. Glatt verdickte interlobuläre Septen können sowohl Zeichen eines Lungenödems als auch einer Fibrose sein. Nodulär verdickte septale Linien sieht man bei einer lymphogenen Metastasierung sowie Sarkoidose [2].

\section{Zystische Veränderungen}

Zysten sind definiert als luftgefüllte Räume, welche von einer relativ dünnen Wand umgeben sind [3, 13]. In der Röntgen-Thorax Aufnahme zeigen sich diese meist eher subtil als eine Aufhellung, manchmal als lineare oder ringförmige Verschattungen oder auch als retikuläres Muster ([2, 14, 15]; - Abb. 3). Die HR-CT zeigt eine deutlich bessere Sensitivität in der Beurteilung von Zysten, welche sich als kreisförmige, zartwandige Aufhellungen, darstellen [3, 

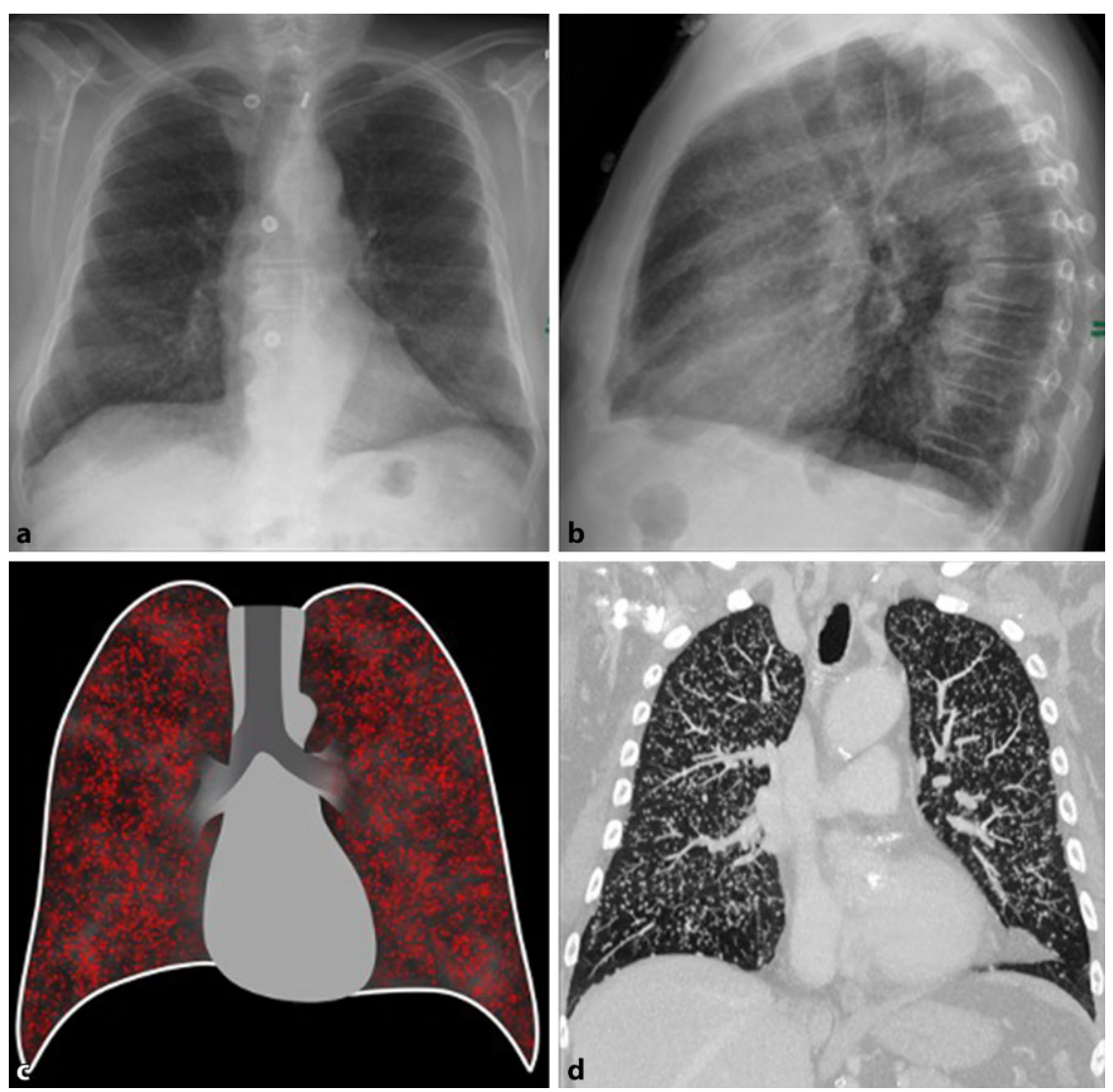

Abb. 5 ム Miliartuberkulose. a, b Thorax-Röntgen p/a und seitliche Aufnahme: Beidseits diffus verteilte mikronoduläre Verdichtungen, cschematische Darstellung der Miliartuberkulose, $d$ HR-CT:zahllose mikronoduläre, diffus über beiden Lungenflügeln verteilte Veränderungen. $H R-C T$ "high resolution" Computertomographie

14]. Eine besondere Form des zystischen Musters ist das Honigwabenmuster, auf Englisch als "honeycombing" bezeichnet. Dieses beschreibt in der CT eine Ansammlung von mindestens zwei oder drei Zysten ähnlichen Durchmessers (3-25 mm) in mehrreihiger Anordnung, und ist das Hauptmerkmal des Musters der gewöhnlichen interstitiellen Pneumonie (UIP), welche sich u.a. in der IPF findet [10, 16]. Die UIP mit Honigwabenmuster ist mit Erkrankungen mit progressiven Verläufen sowie schlechter Prognose wie der IPF vergesellschaftet, weshalb eine frühe Diagnose von fundamentaler Bedeutung ist [16].

Weitere Differentialdiagnosen zystischer Veränderungen beinhalten beispielsweise die Pneumocystis jirovecii Pneumonie, die Langerhans-Zell-Histiozytose (Langerhans cell histiocytosis, LCH), die lymphozytäre interstitielle Pneumonie (LIP), das Birt-Hogg-Dubé Syndrom, die LAM sowie zystische Metastasen [3, 14].

\section{Zusätzliche Befunde}

Zusätzliche radiomorphologische Befunde können weitere Information liefern, welche bei der Erstellung der Differentialdiagnose wichtig sind. Dazu gehören unter anderem Veränderungen des Lungenvolumens, Traktionsbronchiektasien, Lymphadenopathie sowie pleurale Veränderungen [3].

\section{Änderungen des Lungenvolumens} Änderungen des Lungenvolumens finden sich beispielsweise bei der Lungenfibrose als Verlust des Lungenvolumens und beim Lungenemphysem, das durch eine Zunahme des Lungenvolumens gekennzeichnet ist [17].

\section{Traktionsbronchiektasien bzw. Bronchiolektasien}

Die Traktionsbronchiektasien sind definiert als eine irreversible Erweiterung und Verformung der Bronchien und Bron- chiolen in Bereichen von Lungenfibrose. Differentialdiagnostisch kommen dabei beispielsweise die IPF, postradiogene Lungenveränderungen, Asbestose sowie Lungenbeteiligung im Rahmen von Kollagenosen in Frage [3]. Im Röntgen-Thorax ist eine sichere Diagnose von Traktionsbronchiektasen allerdings auf Grund des Überlagerungseffektes meist schwierig [18].

\section{Lymphadenopathie}

Die projektionsradiographisch sichtbare, intrathorakale (z. B. mediastinale und hiläre) Lymphadenopathie ist häufig mit einer Sarkoidose assoziiert (• Abb.4), wobei diese auch bei Silikosen, Berylliosen sowie der LIP auftreten kann [3]. Neben der IPF und $\mathrm{OP}$, findet sich eine mediastinale Lymphadenopathie ebenso manchmal bei NSIPPatienten [19].

\section{Pleurale Veränderungen}

Zu den pleuralen Veränderungen zählen sowohl Pleuraergüsse als auch pleurale Verdickungen. Pleurale Verdickungen findet man bei medikamentenassoziierten interstitiellen Lungerkrankungen oder bei Lungenbeteiligungen im Rahmen von Kollagenosen. Pleuraplaques sind fokale Verbreiterungen der Pleura und sind häufig Folge einer Asbestexposition und können zusammen mit Asbestose (=asbestassoziierte Lungenfibrose) auftreten.

Eine Sonderform des Pleuraergusses ist der Chylothorax. Dieser tritt in 20-40\% der Patienten mit einer LAM auf und ist einer der diagnostischen Kriterien [3]. Obwohl das Thorax-Röntgen eine hohe Sensitivität in der Detektion von Pleuraergüssen bietet, kann es nicht zwischen PleuraergussTypen unterscheiden [20].

\section{Verteilung}

Im letzten Schritt wird die Verteilung des vorgefundenen Musters bestimmt. Dabei unterscheidet man im Thoraxröntgen grundsätzlich folgende Verteilungsmöglichkeiten: 1) kraniokaudale Verteilung, 2) periphere oder zentrale Verteilung [4].

\section{Kraniokaudale Verteilung}

Einige diffuse interstitielle Lungenerkrankungen betreffen überwiegend die 

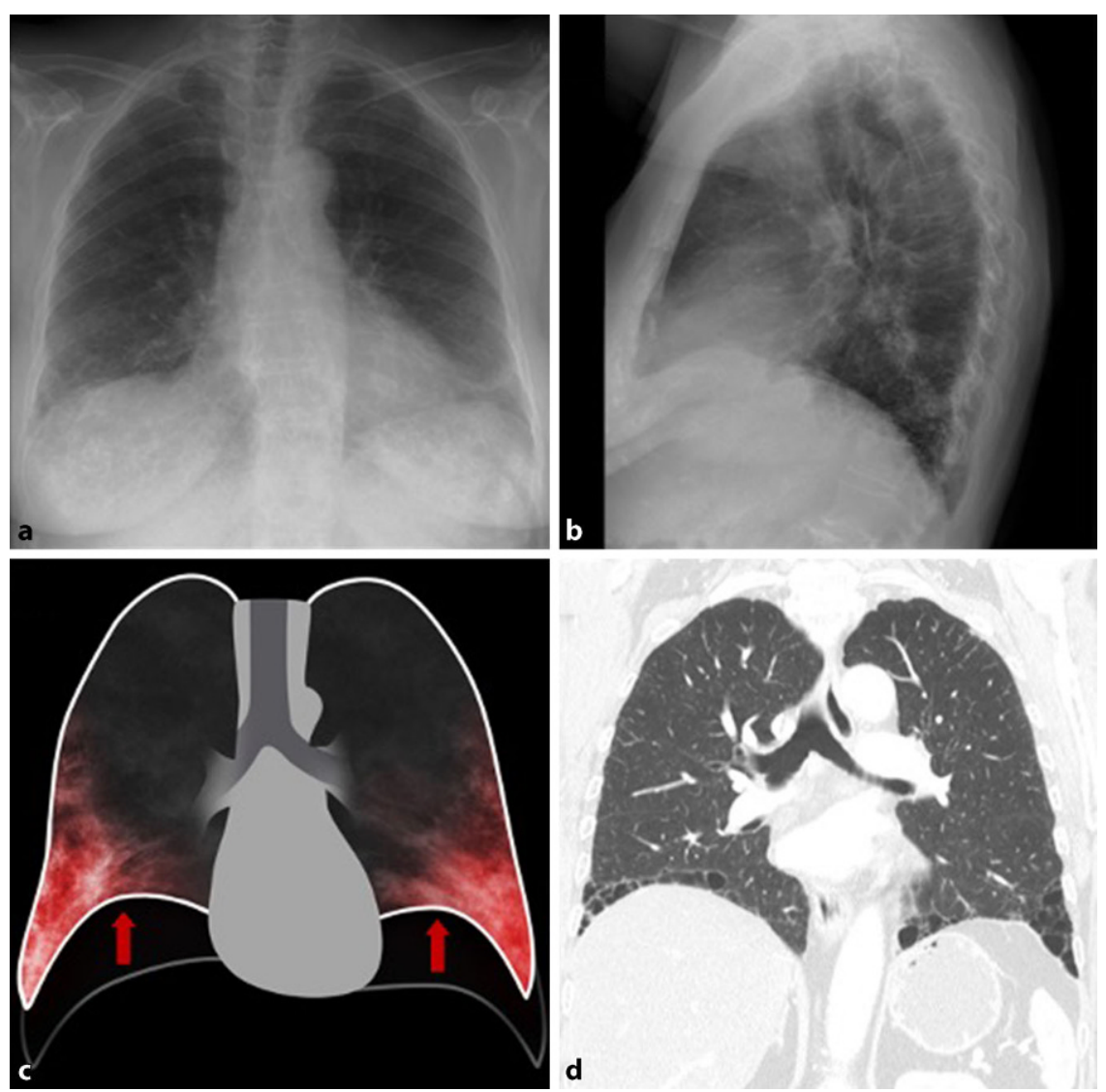

Abb. 6 \ UIP-Muster. a, b Thorax-Röntgen p/a und seitliche Aufnahme: Beidseits dorsobasal betonte Retikulationen mit Zeichen des Volumsverlustes., c schematische Darstellung des unterlappenbetonten UIP Musters mit Volumenverlust (rote Pfeile), d HR-CT: unterlappenbetontes Honeycombing mit geringem Volumenverlust. UIP gewöhnliche interstitielle Pneumonie, $H R-C T$ "high resolution" Computertomographie

Lungen-Oberfelder (z. B. Sarkoidosen, Silikosen, Pneumokoniosen), andere die Lungen-Mittelfelder (z. B. nicht-tuberkulöse Bakterien (NTM)), wohingegen andere eher die Lungen-Unterfelder betreffen (z. B. IPF, NSIP) [4, 21].

\section{Periphere oder zentrale Verteilung}

Eine periphere Verteilung ist charakteristisch für die organisierende Pneumonie, die NSIP oder die UIP. Eine zentrale Verteilung ist typisch für das kardiale Lungenödem oder die pulmonale alveoläre Proteinose [4]. positiver Befund von mikronodulären Veränderungen kann durch interstitielle retikuläre Veränderungen vorgetäuscht werden. In diesem Fall können nur die Kreuzungsstellen der interstitiellen retikulären Veränderungen im Summationsbild sichtbar werden, und somit noduläre Veränderungen vortäuschen [26].

\section{Nicht-infektiöse Erkrankungen}

\section{Idiopathische interstitielle Pneumonien (IIPs)}

Bei den IIPs handelt es sich um eine heterogene Gruppe, die sich radiologisch typischerweise als gewöhnliche interstitielle Pneumonie (UIP), nicht-spezifische interstitielle Pneumonie (NSIP), organisierende Pneumonie (OP), respiratorische Bronchiolitis mit interstitieller Lungenerkrankung (RB-ILD), desquamative interstitielle Pneumonie (DIP), akute interstitielle Pneumonie (AIP), lymphoid interstitielle Pneumonie (LIP) und idiopathische pleuroparenchymale Fibroelastose (PPFE) zeigen [27].

\section{Gewöhnliche interstitielle Pneumonie} (UIP) und Idiopathische pulmonale Fibrose (IPF). Die IPF tritt typischerweise ab einem Alter von 50 Jahren und bevorzugt bei Männern auf [27, 28]. Mit einer Überlebensrate von 2,5-3,5 Jahren gehört diese zu einer Erkrankung mit der schlechtesten Langzeitprognose [27]. Eine positive Raucheranamnese stellt dabei einen Risikofaktor dar. Der Krankheitsbeginn ist schleichend und gekennzeichnet durch eine progrediente Dyspnoe [27, 28]. Das morphologische Muster einer IPF ist die UIP, welche zwar charakteristisch, jedoch nicht spezifisch für die IPF ist [27]. So können differentialdiagnostisch eine EAA, Kollagenosen sowie Vaskulitiden einem UIP-Muster zugrunde liegen [27, 29].

Frühformen der Erkrankung zeigen in der Regel ein unauffälliges Bild im ThoraxRöntgen. Fortgeschrittene Formen hingegen zeichnen sich durch basal betonte, retikuläre Verdichtungen und Volumenverlust, insbesondere der Unterlappen, aus [27]. Als indirekte Zeichen des Volumenverlustes können unter anderem Verziehungen der großen Interlobärsepten sowie hochstehende Zwerchfellkuppeln in beiden Ebenen der Thorax-Röntgen Aufnahme dienen. Die weitere Abklärung 

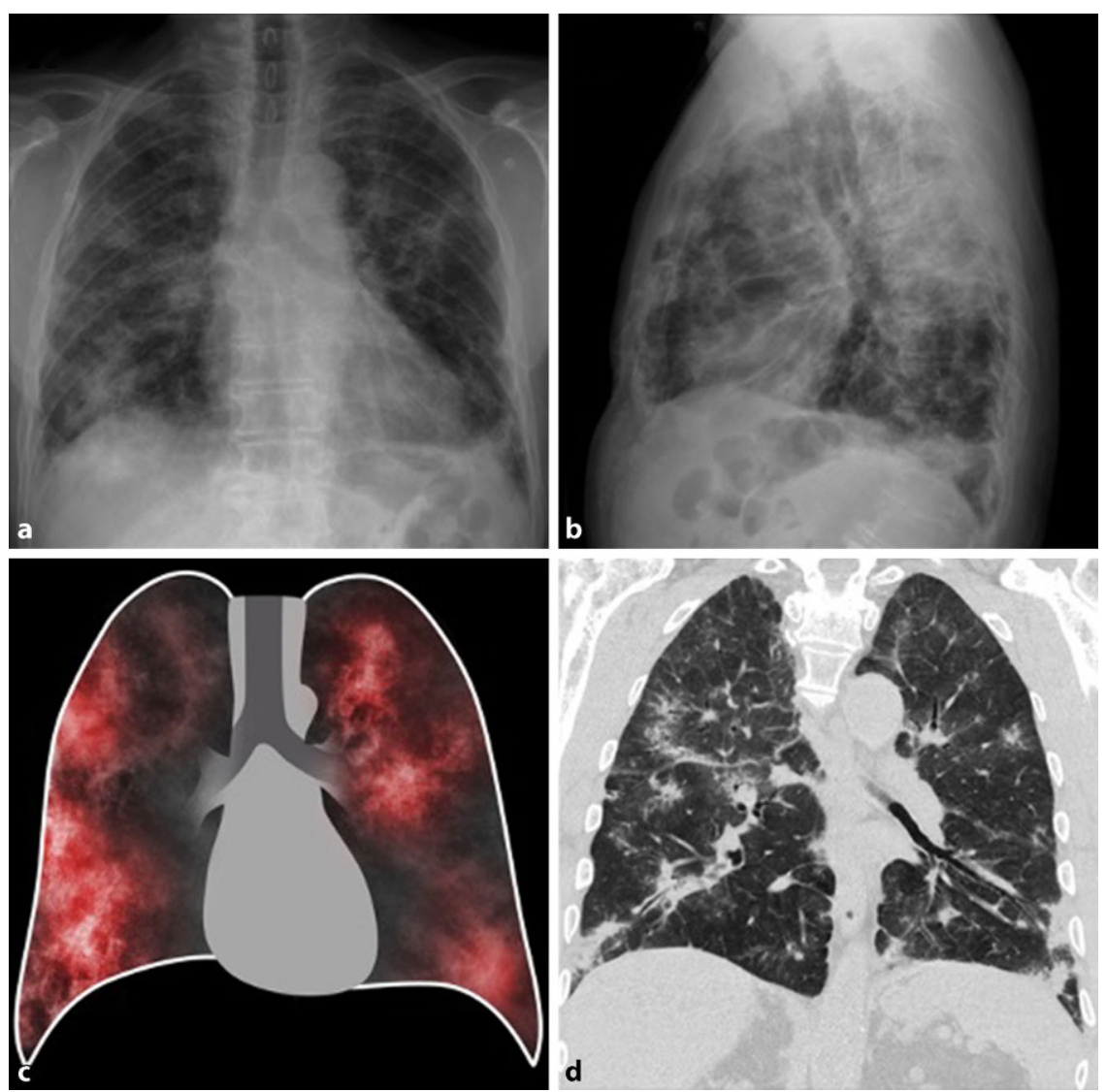

Abb. 7 \ Kryptogene organisierende Pneumonie (COP). a, b Thorax-Röntgen p/a und seitliche Aufnahme: bilateral diffus verteilte, fleckige Verdichtungen, c schematische Darstellung mit peripher betonten fleckigen Transparenzminderungen d HR-CT: subpleural und peribronchial verteilte Konsolidierungen mit Aerobronchogramm und erweiterten Bronchien, mit teilweise Pleurakontakt. $H R$ $C T$,high resolution" Computertomographie

mittels HR-CT ist wesentlich, da im Falle eines typischen UIP-Musters (• Abb. 6), gekennzeichnet von Honeycombing, retikulären Strukturalterationen sowie Traktionsbronchiektasien basaler Betonung sowie dem Fehlen nicht-typischer UIPMuster (z. B. ausgedehnte Milchglasareale, Mikronoduli, Konsolidierungen), mit entsprechender Symptomatik eine weitere Abklärung mittels Biopsie nicht indiziert ist $[27,28]$.

Nicht-spezifische interstitielle Pneumonie (NSIP). Die NSIP, als zweithäufigste IIP nach der UIP, hat einen Altersgipfel von etwa 40-50 Jahren, eine deutlich bessere Prognose und spricht manchmal auf Cortikosteroidtherapie an [27, 30]. Histologisch findet sich bei der NSIP eine homogene Verdickung der alveolären Septen auf Basis von Entzündung oder Fibrose oder einer Kombination aus beiden [31, 32]. Zu Beginn der NSIP zeigt das Thorax-Röntgen keine Auffälligkeiten. In dem fortgeschrittenen Stadium der Erkrankung zeigen sich bilateral fleckige, retikuläre oder noduläre Verdichtungen. Typische NSIP-Veränderungen in der HRCT beinhalten subpleurale, symmetrisch verteilte, meist basal betonte, fleckige, milchglasartige, retikukäre sowie mikronoduläre Verdichtungen. In einem fortgeschrittenen Stadium zeigen sich ebenso Traktionsbronchiektasien sowie Konsolidierungen [30].

Organisierende Pneumonie (OP). Die $\mathrm{OP}$ ist eine überschießende Reparatur von Lungengewebe, das entweder nach Schädigung (z. B. Infektion, Medikamententoxizität, Gasen, Kollagenosen) oder nach Organtransplantation sowie gemeinsam mit Vaskulitiden, malignen Erkrankungen und interstitiellen Lungenerkrankungen auftreten kann [33]. Man spricht von einer kryptogenen Form der OP (cryptoge- ne organisierende Pneumonie, (OP) nur wenn nach ausführliche Abklärung keine andere Ursache dafür gefunden wurde (Auschlussdiagnose) ([33]; 0 Abb. 7).

Im Thorax-Röntgen fallen Konsolidierungen auf, die häufig am Anfang als entzündlich interpretiert werden. In der HR$\mathrm{CT}$ zeigen sich die Veränderungen häufig als subpleural und peribronchial verteilte Konsolidierungen z.T. mit Aerobronchogramm, reversibel erweiterten Bronchien und breitem Pleurakontakt oder (den für die OP suggestiveren) arkardenförmigen Aussparungen subpleural. Milchglas in der Peripherie der Konsolidierungen ist ebenso häufig zu sehen wie eine Migrationstendenz der Konsolidierungen im zeitlichen Verlauf. Ferner sind manchmal bandförmige Konsolidierungen zu sehen, die sich aufgrund von positivem Aerobronchogramm oder aufgrund der kurvenförmigen subpleuralen Anordnung von Plattenatelektasen unterscheiden und neben dem "reverse halo sign" recht charakteristisch sind $[27,33]$.

\section{Desquamative Interstitielle Pneumo-} nie (DIP) und Respiratorische Bronchiolitis mit Interstitieller Lungenerkrankung (RB-ILD). Die DIP repräsentiert den Endzustand der RB-ILD. Obwohl beide Erkrankungen mit einer positiven Raucheranamnese assoziiert sind, kommen differentialdiagnostisch auch Infektionen sowie die Exposition gegenüber organischem Staub als Ursache in Frage [27, 30]. Bei RB-ILD kann das Thorax-Röntgen unauffällig sein, da die Veränderungen subtil sind. In der HR-CT zeigt sich die RB-ILD meist mit zentrilobulären Noduli, Milchglasverschattungen, Bronchialwandverdickungen sowie einem zentroazinären Lungenemphysem. Im Rahmen der DIP sind die Veränderungen im Thorax-Röntgen unspezifisch und zeigen diffuse Verdichtungen. Die HR-CT zeigt bei DIP diffuse milchglasartige Verdichtungen. Zusätzlich können einzelne kleine, scharf berandete Zysten auftreten [30].

Akute interstielle Pneumonie (AIP). Die AIP entspricht dem histologischen Bild eines diffusen Alveolarschadens (DAD) und ist eine akut einsetzende und klinisch rasch fortschreitende interstitielle Pneumonie, die dem klinischen Bild eines 

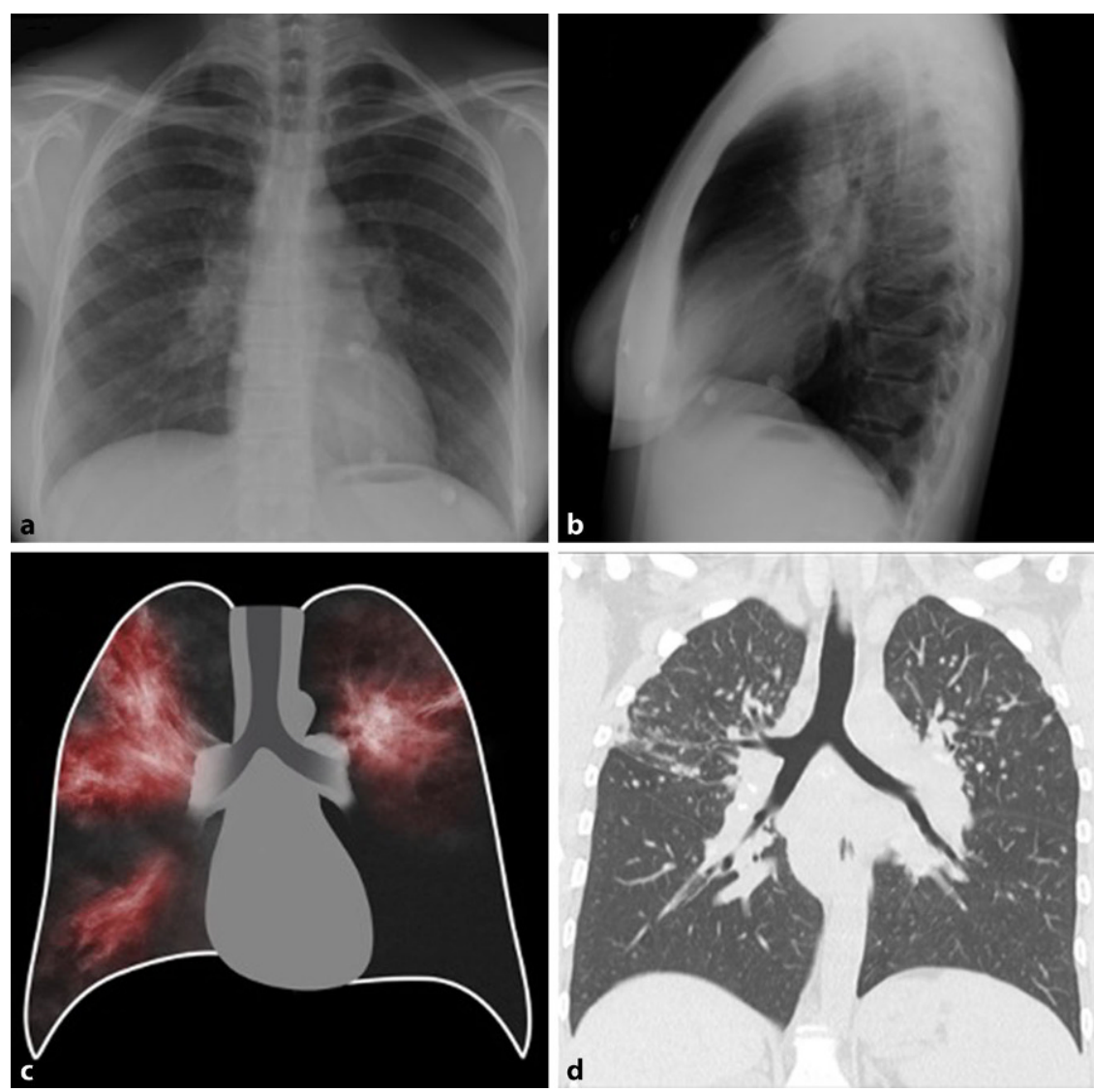

Abb. 8 ॥ Sarkoidose. a, b Thorax-Röntgen p/a und seitliche Aufnahme: bilatrale, hiläre Lymphadenopathie c schematische Darstellung mit bds. perihilären Verändungen und bds. hilärer Lymphadenopathie d HR-CT: mediastinale sowie hiläre Adenopathie bds. mit oberlappenbetonten mikronodulären Veränderungen. $H R-C T$ "high resolution" Computertomographie

ARDS entspricht [27]. Initiale Veränderungen entsprechen peripher betontem Milchglas, das sich mit fortschreitender Erkrankung diffus verteilt und Konsolidierungen bilden kann. Nach Abklingen der akuten Symptomatik können residuäre fibrotische Veränderungen im Sinne von retikulären Verdichtungen mit peripherer und anteriorer Verteilung verbleiben [27].

\section{Lymphoide interstitielle Pneumonie}

(LIP). Die LIP ist die seltenste Form der IIPs und tritt gehäuft in Patienten mit Kollagenosen, HIV-Infektion oder anderer Formen der Immundefizienz auf. Seltener ist die LIP idiopathisch.

Da die typischen pulmonalen Veränderungen in Form von Milchglas mit MosaikMuster und seltener auch Konsolidierungen sehr unspezifisch sind, ist die Anamnese oft wegweisend. Zusätzlich können perivaskuläre, dünnwandige Zysten vorkommen [27].
Pleuroparenchymale Fibroelastose (PPFE). Die PPFE zeichnet sich durch apical und subpleural verteilte Konsolidierungen und miteinhergehender Architekturstörung aus, die sich im Thorax-Röntgen als irreguläre pleuroparenchymale Verdickungen zeigen. Zusätzlich können Traktionsbronchiektasien und Volumenverlust, insbesondere der Oberlappen, beobachtet werden [34].

\section{Sarkoidose}

Bei Patienten mit Sarkoidose ist das Thorax-Röntgen in $90 \%$ der Fälle pathologisch [35]. Zu den typischen Veränderungen im Thorax-Röntgen zählt die symmetrische, bilaterale, hiläre sowie mediastinale (v. a. paratracheale) Lymphadenopathie (- Abb. 8). Der Aspekt der Symmetrie ist hilfreich, um andere Differentialdiagnosen wie Lymphome, Tuberkulose oder Metastasen auszuschließen [8,35]. In $20 \%$ der Fälle zeigen die Patienten eine diffuse Mitbeteiligung des Lungenparenchyms, meist der Lungenoberfelder mit einem retikulonodulären Muster, welches im späteren Verlauf zu einer Fibrose führen kann [8]. Die Thorax-Röntgen basierte Stadieneinteilung der Sarkoidose nach Scadding umfasst 5 Stadien (0 (Normalbefund) bis IV (irreversible Lungenfibrose)) [36]. Bei höherer Sensitivität, zeigt die HR-CT charakteristischerweise gut-definierte mikronoduläre $(2-5 \mathrm{~mm})$ Veränderungen in einer perilymphatischen Verteilung entlang der bronchovaskulären Bündel, der interlobulären Septen und Fissuren sowie subpleurale Veränderungen $[8,35]$.

Langerhans-Zell-Histiozystose (LCH) Die LCH ist eine seltene Erkrankung, welche überwiegend im jungen Erwachsenenalter (20. bis 40. Lj) vor allem bei Rauchern die Lunge betrifft. Oft sind die Symptome sehr mild und unspezifisch können jedoch bis zu einem Pneumothorax reichen. Im Thorax-Röntgen können, neben den oben erwähnten Spontanpneumothoraces, insbesondere symmetrische reticulo-noduläre Veränderungen über beiden Lungenflügeln mit oder ohne zusätzliche zystische Veränderungen, zu sehen sein. Diese finden sich in Lungen mit einem normalen oder sogar erhöhten Lungenvolumen mit Betonung der Mittel- und Oberfelder (• Abb. 9). Lytische Knochenläsionen (vor allem bei Kindern) sollten den Verdacht erhärten. Bei Pleuraergüssen oder hilärer bzw. mediastinaler Lymphadenopathie sollte hingegen an andere Differentialdiagnosen gedacht werden. In weit fortgeschrittenen Fällen können Zeichen einer sekundären pulmonal-arteriellen Hypertonie oder überwiegend zystische Veränderungen des Lungenparenchyms zu sehen sein [37]. In diesem Fall ist zur weiteren Abklärung und gegebenenfalls zur Bestimmung einer Biopsiestelle eine HR-CT des Thorax nahezu unumgänglich. In dieser zeigen sich, getrennt durch normal erscheinendes Lungenparenchym, dick- und dünnwandige Zysten sowie schlecht begrenzte bzw. bizarr konfigurierte noduläre, teils kavernierende Veränderungen (1-15 mm). Die nodulären Veränderungen sind zentrilobulär angeordnet und finden sich diffus verteilt, sparen jedoch meist die basalen Lungenabschnitte aus. Im zeitlichen Verlauf nehmen die nodulären Veränderungen ab und es bleiben dünnwandige 

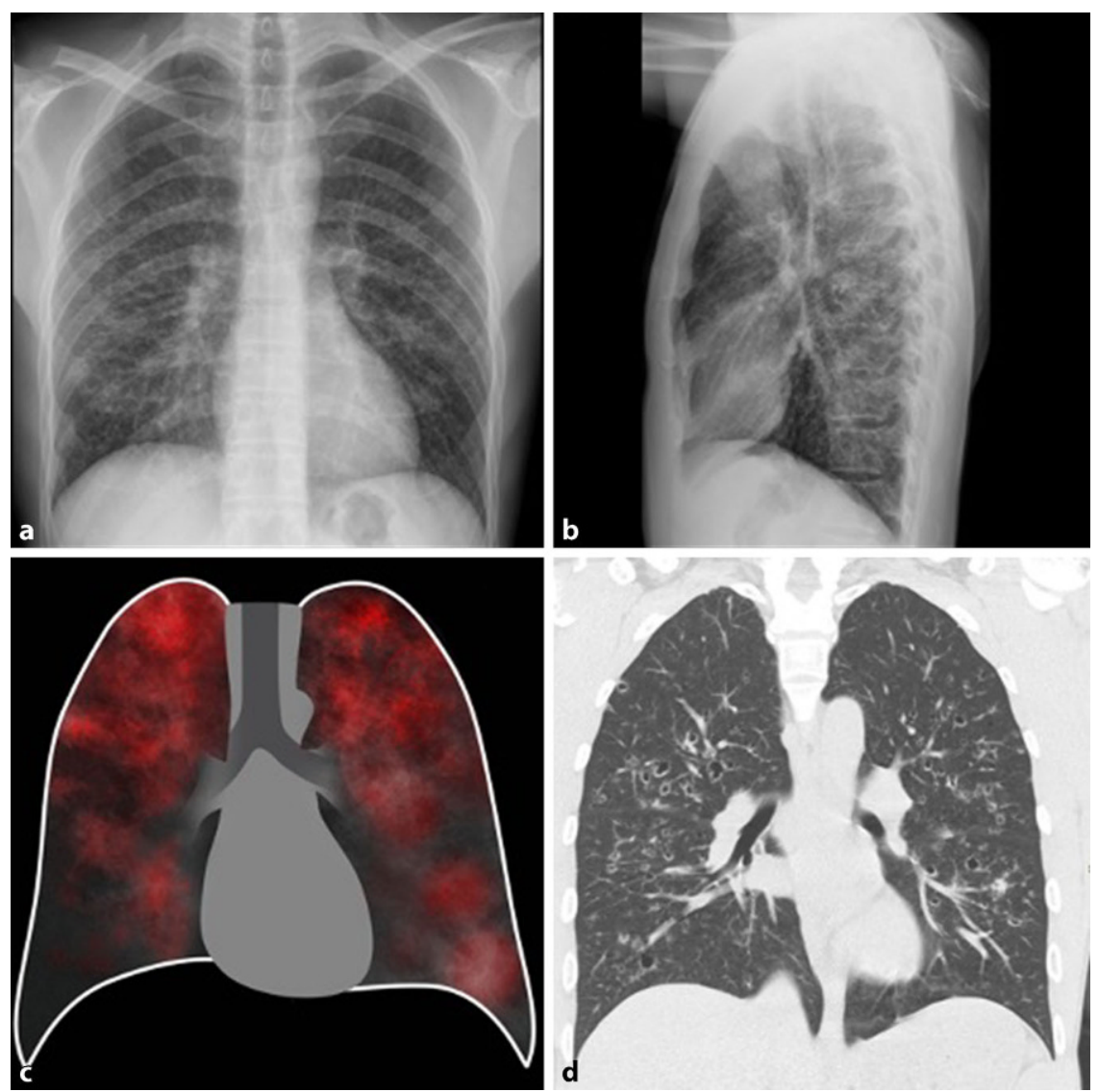

Abb. 9 \ Langerhans-Zell-Histiozytose (LCH). a, b Thorax-Röntgen p/a und seitliche Aufnahme: zystische-noduläre, bilateral diffus verteilte Veränderungen, links mit Betonung des Oberfelds, c schematische Darstellung der teils zystischen Veränderungen mit einer Betonung der Oberlappen, d HRCT: noduläre, zentrilobulär angeordnete, diffus verteilte Veränderungen mit Aussparung der basalen Lungenabschnitte. $H R-C T$ "high resolution" Computertomographie

Zysten $(1-30 \mathrm{~mm})$ bis hin zu einem generalisierten Emphysem als Produkt der vormals kavernierenden Veränderungen zurück $[38,39]$.

\section{Fazit für die Praxis}

- Die Diagnose diffuser parenchymatöser Lungenerkrankungen stellt eine enorme Herausforderung sowohl für Kliniker, Radiologen als auch Pathologen dar und sollte daher bevorzugt im multidisziplinären Rahmen erfolgen.

- Die erste bildgebende Methode bei Patienten, welche mit einer respiratorischen Symptomatik zugewiesen werden, ist das Thorax-Röntgen. Es lassen sich bereits viele Muster (z.B. Milchglasverdichtungen, Konsolidierungen), die Verteilung (kraniokaudal) sowie zusätzliche Befunde (z.B. mediastinale Lymphadenopathie), die auf eine diffuse parenchymatöse Lungenerkrankung hinweisen können, darstellen.

- Die HR-CT („high resolution“ Computertomographie) ist zweifelsfrei ein integraler
Für die aufgeführten Studien gelten die jeweils dort angegebenen ethischen Richtlinien.

\section{Literatur}

1. Lynch J III, Weigt S, Fishbein M (2007) Diffuse parenchymal lung disease. Prog Respir Res 36:11-21

2. Ryu JH et al (2002) Diagnostic approach to the patient with diffuse lung disease. Mayo Clin Proc 77(11):1221-1227 (quiz 1227)

3. Ryu JH et al (2007) Diagnosis of interstitial lung diseases. Mayo Clin Proc 82(8):976-986

4. Nishino M, Itoh $\mathrm{H}$, Hatabu $\mathrm{H}$ (2014) A practical approach to high-resolution $\mathrm{CT}$ of diffuse lung disease. Eur JRadiol 83(1):6-19

5. Wormanns D, Hamer OW (2015) Glossary of terms for thoracic imaging-German version of the Fleischner society recommendations. Rofo 187(8):638-661

6. Diederich S (2010) High resolution computed tomography of the lungs: ground glass opacity and its differential diagnosis. Radiologe 50(12):1141-1152

7. Mueller-Mang C, RinglH, Herold C (2017) Interstitial lung diseases. In: Nikolaou K, Bamberg F, Laghi A, Rubin GD (Hrsg) Multislice CT. Medical radiology. Springer, Cham, S 261-288 https://doi.org/10. 1007/174_2017_151

8. Boitsios G, Bankier AA, Eisenberg RL (2010) Diffuse pulmonary nodules. AJR Am J Roentgenol 194(5):W354-W366

9. Zompatori M et al (2004) Diagnostic imaging of diffuse infiltrative disease of the lung. Respiration 71(1):4-19

10. Marten K (2009) Reticular pattern in thin-section $\mathrm{CT}$ : from morphology to differential diagnosis. Radiologe 49(9):873-881 (quiz 882)

11. Oikonomou A (2014) Role of imaging in the diagnosis of diffuse and interstitial lung diseases. Curr Opin Pulm Med 20(5):517-524

12. Hansell DM et al (2008) Fleischner Society: glossary of terms for thoracic imaging. Radiology 246(3):697-722

13. Ryu JH, Swensen SJ (2003) Cystic and cavitary lung diseases: focal and diffuse. Mayo Clin Proc 78(6):744-752

14. Cantin L, Bankier AA, Eisenberg RL (2010) Multiple cystlike lung lesions in the adult. AJR Am J Roentgenol 194(1):W1-W11

15. Wormanns D, Hamer O (2015) Glossar thoraxradiologischer Begriffe entsprechend der Terminologie der Fleischner Society. Rofo 187(08):638-661

16. Lynch DA et al (2018) Diagnostic criteria for idiopathic pulmonary fibrosis: a Fleischner Society White Paper. Lancet Respir Med 6(2):138-153

17. Tseng $\mathrm{HJ}$ et al (2017) Pulmonary function tests for the radiologist. Radiographics 37(4):1037-1058

18. Westcott JL, Cole SR (1986) Traction bronchiectasis in end-stage pulmonary fibrosis. Radiology 161(3):665-669

19. Souza CA et al (2006) Idiopathic interstitial pneumonias: prevalence of mediastinal lymph node enlargement in 206 patients. AJR Am J Roentgenol 186(4):995-999

20. Majdalany BS et al (2017) ACR Appropriateness Criteria ${ }^{(B)}$ chylothorax treatment planning. J Am Coll Radiol 14(5S):S118-S126

B.H. Heidinger, R.-I. Milos, L. Beer, F. Prayer, S. Röhrich und $H$. Prosch geben an, dass kein Interessenkonflikt besteht.

Für diesen Beitrag wurden von den Autoren keine Studien an Menschen oder Tieren durchgeführt.
21. McDonnell MJ et al (2017) Patterns of disease in patients with middle-lobe predominant bronchiectasis. Respiration 93(6):406-414 
22. Grant LA, Griffin N (2019) Grainger \& Allison's diagnostic radiology essentials, 2. Aufl. Elsevier, Edinburgh

23. Kienzl-PalmaD, ProschH(2016)Thorakale Manifestation der Tuberkulose. Radiologe 56(10):866-873

24. Nachiappan AC et al (2017) Pulmonary tuberculosis:role of radiology in diagnosis and management. Radiographics 37(1):52-72

25. Elicker BM, WebbWR(2013) Fundamentals of highresolution lung CT: common findings, common patterns, common diseases, and differential diagnosis. Wolters Kluwer Health, Lippincott Williams \& Wilkins, Philadelphia, S204-206

26. Voegeli E (2009) Praktische Thoraxradiologie Bd. 5. Huber, Bern, $\mathrm{S} 128$

27. Müller-Mang C et al (2007) Idiopathic interstitial pneumonias: from classification to diagnostic work-up. Radiologe 47(5):384-392

28. Raghu $G$ et al (2018) Diagnosis of idiopathic pulmonary fibrosis. An official ATS/ERS/JRS/ALAT clinical practice guideline. Am J Respir Crit Care Med 198(5):e44-e68

29. Baur X, Fischer A, Budnik LT (2015) Spotlight on the diagnosis of extrinsic allergic alveolitis (hypersensitivity pneumonitis). J Occup Med Toxicol 10:15

30. Mueller-Mang C et al (2007) What every radiologist should know about idiopathic interstitial pneumonias. Radiographics 27(3):595-615

31. Katzenstein AL, Fiorelli RF (1994) Nonspecific interstitial pneumonia/fibrosis. Histologic features and clinical significance. Am J Surg Pathol 18(2):136-147

32. Jegal $Y$ et al (2005) Physiology is a stronger predictor of survival than pathology in fibrotic interstitial pneumonia. Am J Respir Crit Care Med 171(6):639-644

33. Baque-Juston M et al (2014) Organizing pneumonia:What is it? A conceptual approach and pictorial review. Diagn Interv Imaging 95(9):771-777

34. Reddy TLetal (2012) Pleuroparenchymal fibroelastosis: a spectrum of histopathological and imaging phenotypes. Eur Respir J 40(2):377-385

35. Ganeshan D et al (2018) Sarcoidosis from head to toe: what the radiologist needs to know. Radiographics 38(4):1180-1200

36. Scadding JG (1961) Prognosis of intrathoracic sarcoidosis in England. A review of 136 cases after five years' observation. BrMed J 2(5261):1165-1172

37. Tazi A (2006) Adult pulmonary Langerhans' cell histiocytosis. Eur Respir J27(6):1272-1285

38. Schaefer-Prokop C et al (2001) High-resolution CT of diffuse interstitial lung disease: key findings in common disorders. Eur Radiol 11(3):373-392

39. Collins J (2008) Chest radiology: the essentials. Wolters Kluwer, Lippincott Williams \& Wilkins, Philadelphia

\section{Chest radiography findings in diffuse parenchymal lung diseases}

Clinical issue: Diffuse parenchymal lung diseases include a heterogeneous group of diseases of the lung parenchyma, the alveolar spaces, the vessels and the airways, which can be triggered by various pathomechanisms, such as inflammation and fibrotic changes. Since the therapeutic approaches and prognoses differ significantly between the diseases, the correct diagnosis is of fundamental importance. In routine clinical practice, next to the patients' history, the clinical presentation, the laboratory findings and the bronchoscopy, imaging plays a central role in establishing a diagnosis.

Practical recommendations: The diagnosis of diffuse parenchymal lung diseases is an enormous challenge for clinicians, radiologists as well as pathologists and should therefore preferably be carried out in a multidisciplinary setting. Since patients often present with unspecific, respiratory symptoms, chest radiographs are the first imaging method used. Many patterns of diffuse parenchymal lung diseases (e.g., ground-glass opacities and consolidations), their distribution (e.g., cranial-caudal) and the presence of additional findings (e.g., mediastinal lymphadenopathy) are often already detectable on chest X-rays. However, the imaging reference standard and thus, an integral part of the assessment of diffuse parenchymal lung disease, is the chest HR-CT. In some cases, the pattern of the HR-CT is pathognomonic, in others it is unspecific for a disease, so that further diagnostic steps are necessary.

\section{Keywords}

Chest radiography - Diffuse parenchymal lung disease - Idiopathic interstitial pneumonias . Sarcoidosis · Langerhans cell histiocytosis 